\title{
Investigations on the genomic diversity of OXA from isolated Acinetobacter baumannii
}

\author{
Z. Ma ${ }^{1,2}$, L.Q. Zhou ${ }^{3}$, H. Wang ${ }^{4}$ and L.P. Luo ${ }^{1}$ \\ 1'Department of Medical Imaging Center, The First Affiliated Hospital of Jinan University, \\ Guangzhou, China \\ 2Department of Respiratory Diseases, Shenzhen Longgang Central Hospital, \\ Shenzhen, China \\ ${ }^{3}$ Department of Neurosurgery, Shenzhen Longgang Central Hospital, Shenzhen, \\ China \\ ${ }^{4}$ Microbiology laboratory, Shenzhen Longgang Central Hospital, Shenzhen, China \\ Corresponding author: L.P. Luo \\ E-mail: tluolp_jnu@163.com
}

Genet. Mol. Res. 14 (4): 14711-14716 (2015)

Received June 16, 2015

Accepted September 2, 2015

Published November 18, 2015

DOI http://dx.doi.org/10.4238/2015.November.18.36

ABSTRACT. We distinguished the four OXA-type carbapenemase subgroup alleles present in 120 strains of Acinetobacter baumannii by using polymerase chain reaction (PCR) and investigated the distributions of the OXA subgroups in clinically isolated samples. Amplification of the OXA genes blaOXA-23, blaOXA-24, blaOXA-51, and blaOXA-58 was performed by multiplex PCR. Antibiotics susceptibility test was conducted for determine the sensitivity of the $A$. baumannii to clinical common used antibiotics by Kirby-Bauer method. Results revealed that $46(51.69 \%)$ of the samples were positive for only the blaOXA51 gene and $41(46.07 \%)$ were positive for both the blaOXA51 and blaOXA58 genes in the 89 isolates of $A$. baumannii. Among these, 45 were carbapenem-resistant and 44 carbapenem-sensitive. Strains containing either blaOXA51 or blaOXA58 showed resistance or sensitivity to carbapenems, respectively. A. baumannii isolated from intensive care units showed significantly higher resistance rate to Cefepime, Piperacillin-tazobactam, Amikacin, 
Ceftazidime, Cefotaxime, Sulfamethoxazole-trimethoprim, and Gentamicin than those isolated from other departments $(P<0.05)$. In conclusion, we found that the presence of blaOXA-51 and blaOXA-58 appears to convey a mechanism of resistance or sensitivity to carbapenems, respectively, in A. baumannii clinical isolates.

Key words: Genomic diversity; OXA; Acinetobacter baumannii

\section{INTRODUCTION}

Acinetobacter baumannii is an aerobic non-motile gram-negative coccobacillus, and it is considered one of the major nosocomial pathogens worldwide. This polymorphic bacterial pathogen easily spreads between patients and can persist in the environment for several days (Metan et al., 2013). It is well known that $A$. baumannii is the most common pathogenic bacteria isolated from hospitalized patients with pneumonia, other respiratory tract infections, bloodstream infections, urinary tract infections and surgical site infections, and it has an important role in nosocomial infections (Cefai et al., 1990; Lin et al., 2010).

It is reported that Acinetobacter is a common nosocomial pathogen, which is widely observed in intensive care units (ICUs), causing severe infections (Fournier and Richet, 2006). Typically, isolates of $A$. baumannii usually present multidrug resistance, including resistance to third generation cephalosporins, aminoglycosides, and fluoroquinolone (Sinha and Srinivasa, 2007). Previous experimental study has reported that the most common mechanism of drug resistance is the presence of hydrolyzing $\beta$-lactamases, including metallo- $\beta$-lactamases (Ambler class B) and oxacillinases (Ambler class D) (Soares et al., 2012). The widespread use of antimicrobial chemotherapy has played an important role in the appearance of carbapenem-hydrolyzing class D $\beta$-lactamases (CHDLs), which have been widely identified in $A$. baumannii. Four common subgroups of acquired CHDLs are reported in $A$. baumannii, including blaOXA-23, blaOXA-24, blaOXA-51, and blaOXA-58. Here, we set out to distinguish the four OXA subgroup alleles in 120 samples of $A$. baumannii by using multiplex polymerase chain reaction (PCR) and to investigate the distributions of the OXA subgroups in these clinical isolates and the results of antimicrobial susceptibility test.

\section{MATERIAL AND METHODS}

In total, 120 A. baumannii samples were isolated from Shenzhen Longgang Central Hospital between 2010 and 2013. The isolates were identified as $A$. baumannii by a multiple PCR test. The presence of two amplification bands was characteristic of $A$. baumannii. This process allowed 120 samples of $A$. baumannii to be identified, and we randomly selected 89 of these to examine the distributions of OXA gene alleles.

\section{PCR amplification}

Amplification of OXA genes, blaOXA-23, blaOXA-24, blaOXA-51, and blaOXA-58, was performed by multiplex PCR. The primers for blaOXA-23, blaOXA-24, blaOXA-51, and blaOXA-58 utilized were as follows: for blaOXA-23, the forward and reverse primers were 5'-GATCGGATTGGAGAACCAGA-3' and 5'-ATTTCTGACCGCATTTCCAT-3', respectively; for 
blaOXA-24, the forward and reverse primers were 5'-GGTTAGTTGGCCCCCTTAAA-3' and 5'-AGTTGAGCGAAAAGGGGATT-3', respectively; for blaOXA-51, the forward and reverse primers were 5'-TAATGCTTTGATCGGCCTTG-3' and 5'-TGGATTGCACTTCATCTTGG-3', respectively; for blaOXA-58, the forward and reverse primers were 5'-AAGTATTGGGGCTTGTGCTG-3' and 5'-CCCCTCTGCGCTCTACATAC-3', respectively.

\section{Culturing of $A$. baumannii}

The isolates of $A$. baumannii were kept at $-70^{\circ} \mathrm{C}$ until use. Pure cultures of $A$. baumannii were recovered on Mueller-Hinton agar, incubated in a constant temperature incubator at $37^{\circ} \mathrm{C}$. A single colony was cultured in Mueller-Hinton broth (MHB) for $12-16 \mathrm{~h}$ at $37^{\circ} \mathrm{C}$ in a constant temperature incubator, with shaking at $180 \mathrm{rpm}$.

The genomic DNA extraction from all $A$. baumannii isolates was performed with the pure fast bacterial genomic DNA purification kit (Tiangen, Beijing, China). Each PCR reaction mix comprised 50 ng genomic DNA, $200 \mu \mathrm{M}$ dNTPs, $2.5 \mathrm{U}$ Taq DNA polymerase (Promega, Madison, WI, USA), and $200 \mu \mathrm{M}$ primers, in a total volume of $20 \mu \mathrm{l}$. The PCR reactions were initially denatured at $94^{\circ} \mathrm{C}$ for $5 \mathrm{~min}$, followed by 30 cycles of denaturation at $94^{\circ} \mathrm{C}$ for $25 \mathrm{~s}$, and annealing at $53^{\circ} \mathrm{C}$ for $40 \mathrm{~s}$, with a final extension at $72^{\circ} \mathrm{C}$ for $6 \mathrm{~min}$. The PCR products were separated by $1 \%$ agarose gel electrophoresis and visualized using ethidium bromide staining and UV light. Reproducibility was verified by repeating the analysis of a randomly chosen subgroup comprising $10 \%$ of the subject samples.

\section{Antimicrobial susceptibility test}

Antibiotics susceptibility test was conducted for determine the sensitivity of the A. baumannii to clinical common used antibiotics by Kirby-Bauer method (Oxoid Company, Basingstoke, UK). The results were determined by criteria from Clinical and Laboratory Standards Institute (CLSI). The Escherichia coli (ATCC 25922), Pseudomonas aeruginosa (ATCC 27853) and Staphylococcus aureus (ATCC 29213) were used as quality control bacteria.

\section{Statistical analysis}

Statistical analyses were conducted using the SPSS statistical package, version 11.0 (SPSS Inc., Chicago, IL, USA) for Windows. Categorical variables were expressed by frequency and percentage. Differences between categorical variables were compared by $X^{2}$-test. All $P$ values were two sided, and aP value $<0.05$ was considered statistically significant.

\section{RESULTS}

Among the 120 samples, 89 were identified as $A$. baumannii and genotyped for the distribution of the OXA gene alleles. Among the isolated strains, 67 strains $(69.79 \%)$ were isolated from intensive care units, and $29(30.21 \%)$ were from other departments. Of the 89 isolates, 58 $(65.17 \%)$ were isolated from male patients, $31(34.83 \%)$ were from females, $70(78.65 \%)$ were from patients with hospital stays $>4$ weeks.

Multiplex PCR results revealed that of the 89 isolates, 46 (51.69\%) were positive for only the blaOXA51 gene, whereas 41 (46.07\%) were positive for both blaOXA51 and blaOXA58 genes. Two samples (2.25\%) were observed to be positive for both blaOXA51 and blaOXA23 genes. 
However, none of the isolates was positive for the blaOXA-23 and blaOXA-24 combination.

Moreover, we examined resistance to carbapenems in the $A$. baumannii isolates (Table 1). Among the 89 samples, 45 strains (50.56\%) appeared resistant to carbapenems and 44 strains $(49.44 \%)$ showed sensitivity to carbapenems. Among the 46 isolates positive for blaOXA51, we found 8 isolates (8.99\%) with resistance to carbapenems and 38 isolates $(42.70 \%)$ with apparent sensitivity to carbapenems. Of the 41 isolates with both blaOXA51 and blaOXA58, $36(40.45 \%)$ showed resistance to carbapenems, while $5(5.62 \%)$ were sensitive to carbapenems. Of the two strains with both blaOXA51 and blaOXA23, one strain showed resistance $(1.12 \%)$ and the other showed sensitivity to carbapenems (1.12\%).

By Kirby-Bauer method, we compared the drug resistance situation of 89 strains between intensive care units and other departments (Table 2). A. baumannii isolated from intensive care units showed significantly higher resistance rate to Cefepime, Piperacillin-tazobactam, Amikacin, Ceftazidime, Cefotaxime, Sulfamethoxazole-trimethoprim and Gentamicin than those isolated from other departments $(P<0.05)$.

Table 1. Distribution of blaOXA genes in Acinetobacter baumannii clinical isolates and their carbapenem resistance profiles.

\begin{tabular}{lcc}
\hline PCR-positive for genes & Resistant to carbapenems $(\%)$ & Sensitivity to carbapenems $(\%)$ \\
\hline OXA23 & $0(0)$ & $0(0)$ \\
OXA24 & $0(0)$ & $0(0)$ \\
OXA51 & $8(8.99)$ & $41(42.70)$ \\
OXA58 & $0(0)$ & $0(0)$ \\
OXA51+ OXA58 & $38(40.45)$ & $6(5.62)$ \\
OXA51+ OXA23 & $1(1.12)$ & $1(1.12)$ \\
\hline
\end{tabular}

Table 2. Drug resistance situation between groups.

\begin{tabular}{|c|c|c|c|c|}
\hline \multirow[t]{2}{*}{ Antibiotic drugs } & \multicolumn{2}{|c|}{ Resistance rate (\%) } & \multirow[t]{2}{*}{$x^{2}$-test } & \multirow[t]{2}{*}{$P$ value } \\
\hline & In intensive care units & In other departments & & \\
\hline Cefoperazone-sulbactam & 5.62 & 3.37 & 0.26 & 0.61 \\
\hline Meropenem & 21.35 & 10.11 & 1.55 & 0.21 \\
\hline Imipenem & 26.97 & 10.11 & 3.23 & 0.07 \\
\hline Cefepime & 67.42 & 38.20 & 7.12 & 0.01 \\
\hline Piperacillin-tazobactam & 76.40 & 48.31 & 7.18 & 0.01 \\
\hline Amikacin & 78.65 & 51.69 & 7.34 & 0.01 \\
\hline Levofloxacin & 77.53 & 61.80 & 2.48 & 0.12 \\
\hline Ciprofloxacin & 80.90 & 68.54 & 1.55 & 0.21 \\
\hline Tetracycline & 83.15 & 68.54 & 2.62 & 0.11 \\
\hline Ceftazidime & 85.39 & 58.43 & 8.02 & 0.01 \\
\hline Cefotaxime & 86.52 & 61.80 & 7.36 & 0.01 \\
\hline Sulfamethoxazole-trimethoprim & 89.89 & 71.91 & 4.51 & 0.03 \\
\hline Gentamicin & 91.01 & 71.91 & 5.64 & 0.02 \\
\hline Piperacillin & 95.51 & 86.52 & 2.6 & 0.11 \\
\hline
\end{tabular}

\section{DISCUSSION}

Multi-drug resistant $A$. baumannii has caused many nosocomial outbreaks worldwide, and it is considered as a troublesome nosocomial pathogen. Most outbreaks caused by $A$. baumannii present highly resistant to antibiotics, and thus the treatments has become limited (Turton et al., 2006). It is reported that acquired OXA carbapenemases has the main spread of OXA enzymes worldwide.Four common genetic subgroups of acquired CHDLs are reported in $A$. baumannii, 
including blaOXA-23, blaOXA-24, blaOXA-51 and blaOXA-58 (Kusradze et al., 2011; Mohajeri et al., 2013; Cayô et al., 2014; Aly et al., 2014).

In our study, we only detected positive samples for blaOXA-51 and blaOXA-58, known to be distributed globally among $A$. baumannii isolates; furthermore, we found that blaOXA-51 and blaOXA-58 appear to be important in conferring resistance or sensitivity to carbapenems, respectively. In the present study, we found that 46 (51.69\%) were positive for only the blaOXA51 gene, whereas $41(46.07 \%)$ were positive for both blaOXA51 and blaOXA58 genes. Two samples $(2.25 \%)$ were observed to be positive for both blaOXA51 and blaOXA23 genes. The results of our study show similarities with those of previous studies (Ciftci et al., 2010; Amudhan et al., 2011; Bali et al., 2013; Mohajeri et al., 2013). In a recent study, Ciftci et al. (2010) identified the frequencies of blaOXA23, blaOXA51 and blaOXA58 genes in carbapenem-resistant A.baumannii clinical isolates. Amudhan et al. (2011) reported that blaOXA-23 and blaOXA 51 genes are the most common types of OXA carbapenamases. Bali et al. (2013) reported that blaOXA-23 and blaOXA-51 were the major factors underlying the pathogenicity for carbapenem-resistant Acinetobacter. Mohajeri et al. (2013) reported that blaOXA-51-like and blaOXA-23-like genes were the predominant mechanisms of resistance to imipenem in $A$. baumannii. However, certain results of previous studies appear to be inconsistent with those of our study (Jeannot et al., 2014; Ji et al., 2014; Xia et al., 2014; Mohajeri et al., 2015). One study reported that only blaOXA-23 and blaOXA-51 were amplified in $96.7 \%$ of the A. baumannii strains, while neither blaOXA-24 nor blaOXA-58 was amplified (Xia et al., 2014). Jeannot et al. (2014) reported that blaOXA-23 and blaOXA-24 were the most frequently detected genes in isolated $A$. baumannii. Ji et al. (2014) reported that blaOXA-24 and blaOXA-58 have emerged as potential threats for hospital outbreaks of multidrug-resistant $A$. baumannii. Mohajeri et al. (2015) observed that blaOXA-23-like gene were the main genes of $A$. baumannii. for high resistant to anti-mocrobial agents. The discrepancies between the studies may be due to differences in sample selection or genetic variation among the different ethnicities examined, as well as the sample size used for the study. Therefore, further studies are pressingly needed to confirm our findings.

Our study found that the $A$. baumannii isolated from intensive care units showed significantly higher resistance rate to Cefepime, Piperacillin-tazobactam, Amikacin, Ceftazidime, Cefotaxime, Sulfamethoxazole-trimethoprim and Gentamicin than those isolated from other departments. The main mechanism of drug resistant from $A$. baumannii is caused by the four carbapenemases. OXA23, OXA-24, OXA-51, and OXA-58 belongs to the carbapenemases.

In summary, we have suggested that the presence of blaOXA-51 and blaOXA-58 is the main mechanism of resistance or sensitivity to carbapenems, respectively. Both phenotyping and genotyping methods are important for the detection of carbapenem resistance in Acinetobacter.

\section{Conflicts of interest}

The authors declare no conflict of interest.

\section{REFERENCES}

Alvargonzalez JJ, Vindel HA, Martín MD, Casas CM, et al. (2014). Sequential outbreaks in a Spanish hospital caused by multiresistant OXA-58-producing Acinetobacter baumannii of ST92. J. Med. Microbiol. 63: 1093-1098.

Aly M, Tayeb HT, Al Johani SM, Alyamani EJ, et al. (2014). Genetic diversity of OXA-51-like genes among multidrug-resistant Acinetobacter baumannii in Riyadh, Saudi Arabia. Eur. J. Clin. Microbiol. Infect. Dis. 33: 1223-1228.

Bali NK, Fomda BA, Bashir H, Zahoor D, et al. (2013). Emergence of carbapenem-resistant Acinetobacter in a temperate north Indian State. Br. J. Biomed. Sci. 70: 156-160. 
Cayô R, Merino M, Ruiz Del Castillo B, Cano ME, et al. (2014). OXA-207, a novel OXA-24 variant with reduced catalytic efficiency against carbapenems in Acinetobacter pitti from Spain. Antimicrob. Agents. Chemother. 58: 4944-4948.

Cefai C, Richards J, Gould FK and McPeake P (1990). An outbreak of Acinetobacter respiratory tract infection resulting from incomplete disinfection of ventilatory equipment. J. Hosp. Infect. 15: 177-182.

Fournier PE and Richet H (2006). The epidemiology and control of Acinetobacter baumannii in health care facilities. Clin. Infect. Dis. 42: 692-699.

Jeannot K, Diancourt L, Vaux S, Thouverez M, et al (2014). Molecular epidemiology of carbapenem non-susceptible Acinetobacter baumannii in France. PLoS One. 9: e115452.

Ji S, Chen Y, Ruan Z, Fu Y, et al. (2014). Prevalence of carbapenem-hydrolyzing class D $\beta$-lactamase genes in Acinetobacter spp. isolates in China. Eur. J. Clin. Microbiol. Infect. Dis. 33: 989-997.

Kusradze IA, Diene SM, Goderdzishvili M and Rolain JM (2011). Molecular detection of OXA carbapenemase genes in multidrug-resistant Acinetobacter baumannii isolates from Iraq and Georgia. Int. J. Antimicrob. Agents. 38: 164-168.

Lin YC, Sheng WH, Chen YC, Chang SC, et al. (2010). Differences in carbapenem resistance genes among Acinetobacter baumannii, Acinetobacter genospecies 3 and Acinetobacter genospecies 13TU in Taiwan. Int. J. Antimicrob. Agents. 35: 439-443.

Metan G, Sariguzel F, Sumerkan B, Reijden TY, et al. (2013). Clonal diversity and high prevalence of OXA-58 among Acinetobacter baumannii isolates from blood cultures in a tertiary care centre in Turkey. Infect. Genet. Evol. 14: 92-97.

Mohajeri P, Farahani A, Feizabadi MM, Ketabi H, et al. (2013). Antimicrobial susceptibility profiling and genomic diversity of Acinetobacter baumannii isolates: A study in western Iran. Iran. J. Microbiol. 5: 195-202.

Mohajeri P, Farahani A, Feizabadi MM, Norozi B (2015). Clonal evolution multi-drug resistant Acinetobacter baumannii by pulsed-field gel electrophoresis. Indian. J. Med. Microbiol. 33: 87-91.

Sinha M and Srinivasa H (2007). Mechanisms of resistance to carbapenems in meropenem-resistant Acinetobacter isolates from clinical samples. Ind. J. Med. Microbiol. 25: 121-125.

Soares GM, Figueiredo LC, Faveri M, Cortelli SC, et al. (2012). Mechanisms of action of systemic antibiotics used in periodontal treatment and mechanisms of bacterial resistance to these drugs. J. Appl. Oral. Sci. 20: 295-309.

Turton JF, Ward ME, Woodford N, Kaufmann ME, et al. (2006). The role of ISAba1 in expression of OXA carbapenemase genes in Acinetobacter baumannii. FEMS Microbiol. Lett. 258: 72-77.

Xia J, Zhang D, Xu Y, Gong M, et al. (2014). A retrospective analysis of carbapenem-resistant Acinetobacter baumannii-mediated nosocomial pneumonia and the in vitro therapeutic benefit of cefoperazone/sulbactam. Int. J. Infect. Dis. 23: 90-93. 